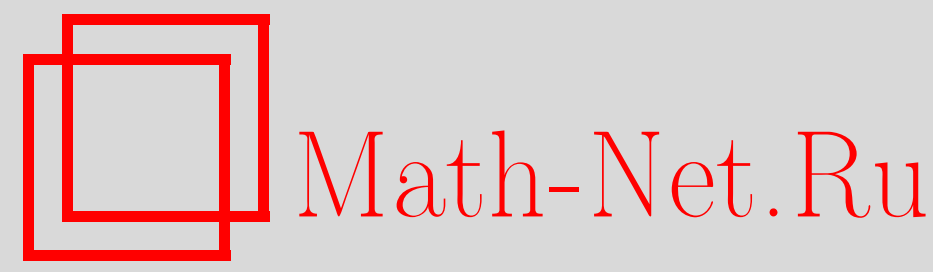

В. А. Садовничий, В. Е. Подольский, Об одном классе операторов ШтурмаЛиувилля и приближенном вычислении первых собственных значений, Матем. сб., 1998, том 189, номер 1, 133-148

DOI: https://doi.org/10.4213/sm298

Использование Общероссийского математического портала Math-Net.Ru подразумевает, что вы прочитали и согласны с пользовательским соглашением

http://www . mathnet.ru/rus/agreement

Параметры загрузки:

IP : 52.87 .193 .239

26 апреля 2023 г., 16:06:40 
УДК 517.94

\author{
В. А. Садовничий, В.Е. Подольский
}

\title{
Об одном классе операторов Штурма-Лиувилля и приближенном вычислении первых собственных значений
}

\begin{abstract}
В работе определяется и исследуется специальный класс $S$ операторов Штурма-Лиувилля с простьми асимптотическими свойствами собственных функций. Изучены аналитические свойства потенциалов и дано описание операторов этого класса в терминах переходной функции обратной задачи. Доказана теорема: класс операторов $S$ плотен в множестве операторов IIтурма-Лиувилля $с$ потенциалом из $L_{2}$. Далее выделено явно конструируемое подмножество операторов класса $S$, сохраняющее свойство плотности, и на основании свойств операторов этого подмножества предложен и обоснован метод приближенного вычисления первых собственных чисел оператора Штурма-Лиувилля из его регуляризованных следов

Библиографияя: 13 названий.
\end{abstract}

1. Введение. Задача о вычислении первых собственных чисел оператора Штурма-Лиувилля является одной из важных классических задач математической физики. Достаточно упомянуть ее роль в использовании метода Фурье для приближенного решения классических краевых задач в частных производных и в задачах теории устойчивости. Естественно, что проблеме вычисления первых собственных чисел посвящены исследования многих математиков. Мы сосредоточимся на обсуждении теоретических методов и алгоритмов, не касаясь методов численных.

Одним из наиболее употребительных способов является метод, основанньй на хорошо известных равенствах, связьвающих итерированные функции Грина рассматриваемой задачи и ее собственные значения:

$$
\int_{0}^{\pi} G_{k}(x, x) d x=\sum_{n=0}^{\infty} \frac{1}{\lambda_{n}^{k}}, \quad k=1,2, \ldots
$$

Одно из наиболее глубоких исследований в этом направлении принадлежит А.А. Дородницыну [1]. Суть метода проста: обрываем в равенствах (1) ряды до слагаемых с номером $N$ и берем первые $(N+1)$-но первое равенство. Решаем полученную конечную систему и получаем приближенные значения собственных чисел, тем более точные, чем большее $N$ взято. В силу хорошо известной во многих классических задачах локализации собственных чисел с точностью до $O(1)$ оценку отброшенного остатка ряда сделать несложно и корректность метода очевидна.

Работа вьполнена при поддержке Российского фонда фундаменталњных исследований (грант ведущих научных школ № 96-15-96049). 
Вместе с тем метод обладает существенным недостатком: вычисление конкретных значений интегралов из левой части (1) не алгоритмизуется и эти интегралы в конечном виде через параметры исходной задачи, вообще говоря, не выражаются.

С созданием теории регуляризованных следов И. М. Гельфандом и Б. М. Левитаном [2] в 1953 г. и результатами И.М. Гельфанда [3], Л.А. Дикого [4], а также с появлением фундаментальной работы В.Б. Лидского и В.А. Садовничего [5] в распоряжении математиков появились новые соотношения на собственные числа операторов - регуляризованные следы $k$-го порядка:

$$
\sum_{n=0}^{\infty}\left[\lambda_{n}^{k}-A_{n}(k)\right]=B(k), \quad k=1,2, \ldots
$$

здесь $A_{n}(k)$ - отрезок асимптотического разложения $\lambda_{n}^{k}$ по степеням $n$ такой, что обеспечена абсолютная сходимость ряда (но, вообще говоря, не обязательно минимальный из таких фрагментов асимптотического разложения), а $B(k)$ - сумма этого ряда, собственно и называемая $k$-ым регуляризованньм следом. Эти равенства важны и интересны из-за того, что и $A_{n}(k)$ и $B(k)$ выражаются в конечном виде через коэффициенты дифференциального выражения и краевых условий и их вычисление вполне может быть алгоритмизировано (см. [5], [6]).

В связи с этим И.М. Гельфанд и Л.А. Дикий [7] предложили в 1957 г. новый метод приближенного вычисления первых собственных чисел оператора Штурма-Лиувилля: аналогично схеме использования системы (1) удержать в системе (2) частные суммы рядов до $N$-го слагаемого в $(N+1)$-ом регуляризованном следе и полученную приближенную систему решить, найдя некоторые приближения к собственным числам задачи. В [7] сделано конкретное вычисление для уравнения Матье по указанной схеме и получены значения трех первых собственных значений, верные в третьем знаке после запятой. Однако в [7] данный метод не обоснован: никаких оценок при переходе от рядов к их частньм суммам сделано не было.

В 1995 г. С.А. Шкарин [8] доказал неединственность решения бесконечных линейных систем определенного вида и, в частности, для систем вида (2) из его результатов следует, что если в системе регуляризованных следов (2) числа $\lambda_{n}$ считать неизвестными и решать эту систему относительно $\lambda_{n}$, то у (2) существует континуум решений, причем мы можем заранее совершенно произвольно задать любое конечное число (различных) собственных чисел и всегда существуют решения с этими заданньми числами. Таким образом, было показано, что метод приближенного вычисления первых собственных чисел с помощью системы регуляризованных следов в трактовке И. М. Гельфанда и Л.А. Дикого не может быть реализован.

В настояшей работе ${ }^{1}$ мы определяем и исследуем класс $S$ операторов Штурма-Лиувилля, для которых система регуляризованных следов однозначно определяет все их собственные числа и позволяет приближенно вычислять первые. В числе доказанных свойств этого класса операторов есть следующее: этот класс плотен в множестве всех операторов Штурма-Лиувилля с потенциалом из $L_{2}$ в смысле операторной нормы. Эти два результата образуют метод приближенного вычисления первых собственных чисел любого оператора с потенциалом из $L_{2}$ : сначала

\footnotetext{
${ }^{1}$ Эти результаты мы анонсировали в работе [9]
} 
мы должны приблизить рассматриваемый оператор (методом, изложенным в п. 4) с точностью до $\varepsilon / 2$ оператором из класса $S$ и затем уже для построенного приближающего оператора найти с точностью до $\varepsilon / 2$ первые собственные числа (методом, изложенным в п. 3). Таким образом, собственные числа исходного оператора будут найдены с точностью до $\varepsilon$.

2. Операторы класса $S$. Рассмотрим оператор Штурма-Лиувилля

$$
\begin{gathered}
-y^{\prime \prime}+q(x) y=\lambda y, \\
y^{\prime}(0)-h y(0)=0, y^{\prime}(\pi)+H y(\pi)=0,
\end{gathered}
$$

где $q(x) \in L_{2}[0, \pi], h, H \in \mathbb{R}$. На протяжении всей работы мы будем обозначать через $\varphi(x, \lambda)$ решение задачи Коши для уравнения $(3)$ с начальньми данными $\varphi(0, \lambda)=1, \varphi^{\prime}(0, \lambda)=h$.

Если $q(x) \in C^{n}[0, \pi]$, то хорошо известно (см., например, [10]), что $\varphi(x, \lambda)$ при $|\lambda| \rightarrow \infty$ имеет следующее асимптотическое разложение:

$$
\begin{aligned}
\varphi(x, \lambda)=\cos \sqrt{\lambda} x+k_{1}(x) \frac{\sin \sqrt{\lambda} x}{\sqrt{\lambda}} & +\cdots+k_{2 i}(x) \frac{\cos \sqrt{\lambda} x}{(\sqrt{\lambda})^{2 i}} \\
& +k_{2 i+1} \frac{\sin \sqrt{\lambda} x}{(\sqrt{\lambda})^{2 i}}+\cdots+O\left(\frac{e^{|\Im \lambda| \pi}}{|\lambda|^{\frac{n+1}{2}}}\right),
\end{aligned}
$$

где $k_{i}(x)$ можно найти из следуюшей рекуррентной цепочки соотношений:

$$
\begin{array}{rlrl}
k_{1}^{\prime}(x) & =\frac{1}{2} q(x), & k_{1}(0) & =h, \\
k_{2}^{\prime}(x) & =\frac{1}{2}\left(k_{1}^{\prime \prime}(x)-q(x) k_{1}(x)\right), & k_{2}(0)=0 \\
k_{3}^{\prime}(x) & =\frac{1}{2}\left(-k_{2}^{\prime \prime}(x)+q(x) k_{2}(x)\right), & k_{3}(0)=-k_{2}^{\prime}(0), \\
\ldots \ldots \ldots \ldots \ldots \ldots \ldots \ldots \ldots \ldots \ldots \ldots \ldots \ldots \ldots \ldots \ldots \ldots \ldots \ldots \ldots \ldots \ldots \ldots \ldots \ldots \ldots \ldots & \\
k_{l}^{\prime}(x) & =\frac{(-1)^{l+1}}{2}\left(-k_{l-1}^{\prime \prime}(x)+q(x) k_{l-1}(x)\right) \\
& k_{2 i}(0)=0, \quad k_{2 i}^{\prime}(0)+k_{2 i+1}(0)=0
\end{array}
$$

Дадим следующее важное

ОПРЕДЕЛЕНИЕ. Оператор вида (3)-(4) мы назовем оператором класса $S[0, \pi]$, если в разложении (5) для некоторого целого $j, 1 \leqslant j \leqslant n+1, k_{j}(x) \equiv 0$ на $[0, \pi]$.

ЗАмечАниЕ. Аналогично можно дать определение операторов класса $S[0, a]$ для любого вещественного $a$. Мы часто будем писать просто $S$ без указания отрезка, когда это не может привести к недоразумению.

Данное определение автоматически подразумевает наличие у потенциала соответствующей гладкости.

Далее мы изучим некоторые свойства операторов класса $S$. 
ЛЕмма 1. Потенииал $q(x)$ любого оператора класса $S$ есть голоморфная в некоторой окрестности отрезка $[0, \pi]$ функиия, не продолжсающаяся на всю плоскость как целая.

ДокАЗАтЕльство. Пусть для некоторого номера $N k_{N}(x) \equiv 0$. Тогда в формулах (6) имеем

$$
\begin{gathered}
k_{N}^{\prime}(x) \equiv 0=\frac{(-1)^{N+1}}{2}\left(-k_{N-1}^{\prime \prime}(x)+q(x) k_{N-1}(x)\right), \\
k_{N+1}^{\prime}(x)=\frac{(-1)^{N+2}}{2}\left(-k_{N}^{\prime \prime}(x)+q(x) k_{N}(x)\right) \equiv 0 .
\end{gathered}
$$

Причем если $N+1$ четное, то $k_{N+1}(0)=0$ и тогда $k_{N+1}(x) \equiv 0$, а если $N+1$ нечетное, то $k_{N}^{\prime}(0)+k_{N+1}(0)=0$, отсюда $k_{N+1}(0)=0$ и все равно $k_{N+1}(x) \equiv 0$. Таким образом, из (8) и (6) следует, что если $k_{N}(x) \equiv 0$, то для любого $n>N$ $k_{n}(x) \equiv 0$ и $(7)$ - последнее нетривиальное соотношение в цепочке равенств (6). Обозначив $k_{l}^{\prime}(x)=k_{l}^{*}(x)$, мы получаем из (6) систему уравнений первого порядка относительно функций $k_{l}(x), k_{l}^{*}(x), l=1, \ldots, N$, разрешенную относительно производных, содержащую $2 N$ у равнений с полиномиальными нелинейностями без явного вхождения независимой переменной. Из этой системы возможно исключить все неизвестные, кроме $k_{1}^{*}(x)$ (т.е. $q(x)$ ), и полученное нелинейное дифференциальное уравнение удовлетворяет условиям следуюшей теоремы [10, с. 116]: если в дифференциальном уравнении $P\left(z, w, w^{\prime}, \ldots, w^{(n)}\right)=0$

$$
P=\sum a_{j_{0} j_{1} \ldots j_{n}}(z) w^{j_{0}}\left(w^{\prime}\right)^{j_{1}} \cdots\left(w^{(n)}\right)^{j_{n}}
$$

есть многочлен по всем переменным и $d=\max \left(j_{0}+\cdots+j_{n}\right)$ достигается только на одном слагаемом, то у уравнения нет целых трансцендентных решений. Заметив к этому, что из (6) в случае, когда $q(x)$ многочлен, следует, что и все $k_{j}(x)$ многочлены строго возрастающих степеней и, следовательно, не может быть обрыва асимптотического разложения (5), мы приходим к выводу, что $q(x)$ не целая. Так как $q(x)$ непрерьвна на отрезке $[0, \pi]$, то в некоторой окрестности этого отрезка полюсов у $q(x)$ нет. Лемма доказана.

Для дальнейшего необходимо напомнить некоторые определения и факты из обратной задачи для оператора Штурма-Лиувилля.

Оператором преобразования для оператора (3)-(4) и оператора того же вида с $q(x) \equiv 0$ и $h=0$ называется обратимый оператор вида $I+K$, где $K$ - интегральньй оператор с треугольным ядром $K(x, y)$, переводящий $\cos \sqrt{\lambda} x$ в $\varphi(x, \lambda)$ :

$$
\varphi(x, \lambda)=\cos \sqrt{\lambda} x+\int_{0}^{x} K(x, t) \cos \sqrt{\lambda} t d t, \quad 0 \leqslant x \leqslant \pi .
$$

Известно (см., например, [12]), что $K(x, y)$ является решением следующей задачи:

$$
\begin{gathered}
\frac{\partial^{2} K}{\partial x^{2}}+q(x) K=\frac{\partial^{2} K}{\partial y^{2}} \\
K_{y}^{\prime}(x, 0)=0, \quad K(x, x)=h+\frac{1}{2} \int_{0}^{x} q(t) d t
\end{gathered}
$$

и если известна функция $K(x, y)$, то $q(x)=2 \frac{d}{d x} K(x, x), h=K(0,0)$. 
Переходной функцией обратной задачи $\Phi(x)$ называется следуюшая функция, строящаяся по заданному спектру оператора $\left\{\lambda_{n}\right\}$ и заданным нормировочным числам $\left\{\alpha_{n}, \alpha_{n}=\int_{0}^{\pi} \varphi^{2}\left(x, \lambda_{n}\right) d x\right\}$ :

$$
\Phi(x)=\sum_{n=0}^{\infty}\left(\frac{1}{\alpha_{n}} \cos \sqrt{\lambda_{n}} x-\frac{2}{\pi} \cos n x\right)+\frac{1}{\pi} .
$$

Основное интегральное уравнение обратной задачи (уравнение Гельфанда-Левитана) связывает $K(x, y)$ и $\Phi(x)$ :

$$
K(x, y)+F(x, y)+\int_{0}^{x} K(x, t) F(t, y) d t=0, \quad 0 \leqslant y \leqslant x \leqslant \pi
$$

где

$$
F(x, y)=\frac{1}{2}[\Phi(x+y)+\Phi(|x-y|)]
$$

Мы можем сформулировать следующую лемму.

Лемма 2. Если потенциал $q(x)$ оператора (3)-(4) аналитичен в некоторой окрестности отрезка $[0, \pi]$, то ядро оператора преобразования $K(x, y)$ аналитично по каждой переменной в некоторой окрестности отрезка $[0, \pi]$ и переходная функция обратной задачи $\Phi(x)$ аналитична в некоторой окрестности отрезка $[0,2 \pi]$.

ДокАЗАтЕльство. Сделав в задаче (10) замену переменных $x=\frac{1}{2}(\alpha+\beta)$, $y=\frac{1}{2}(\alpha-\beta)$ и обозначив $M(\alpha, \beta)=K\left(\frac{\alpha+\beta}{2}, \frac{\alpha-\beta}{2}\right)$, можно получить для функции $M(\alpha, \beta)$ следуюшее интегральное уравнение (см. $[12$, с. 16,17$])$ :

$$
\begin{aligned}
M(\alpha, \beta)=h+\frac{1}{4} \int_{\beta}^{\alpha} & q\left(\frac{\delta}{2}\right) d \delta+\frac{1}{4} \int_{\beta}^{\alpha} d \delta \int_{0}^{\beta} q\left(\frac{\delta+\gamma}{2}\right) M(\delta, \gamma) d \gamma \\
& +\frac{1}{2} \int_{0}^{\beta} q\left(\frac{\delta}{2}\right) d \delta+\frac{1}{2} \int_{0}^{\beta} \int_{0}^{\delta} q\left(\frac{\delta+\gamma}{2}\right) M(\delta, \gamma) d \gamma d \delta
\end{aligned}
$$

Уравнение (4) есть уравнение типа Вольтерра, оно может быть решено с помощью метода последовательных приближений, причем последовательность этих приближений будет сходиться к решению равномерно на интересующей нас области. Отсюда следует, что если $q(x)$ - аналитическая функция, то функция $K(x, y)$ также есть аналитическая функция.

Далее, интегральное уравнение обратной задачи (12), рассмотренное как уравнение относительно неизвестной функции $F(x, y)$, есть уравнение с независимой переменной $x$ и параметром $y$. При значении параметра $y=0$ это уравнение переходит в уравнение типа Вольтерра относительно переходной функции $\Phi(x)$ :

$$
K(x, 0)+\Phi(x)+\int_{0}^{x} K(x, t) \Phi(t) d t=0 .
$$


Отсюда сразу следует, что при аналитической $K(x, y)$ функция $\Phi(x)$ также аналитична при $0 \leqslant x \leqslant \pi$. Для доказательства ее аналитичности при $0 \leqslant x \leqslant 2 \pi$ рассмотрим уравнение (12) при $y=\pi / 2$ :

$$
\begin{aligned}
& {\left[K\left(x, \frac{\pi}{2}\right)+\frac{1}{2} \Phi\left(x-\frac{\pi}{2}\right)\right.} \\
& \left.\quad+\frac{1}{2} \int_{0}^{\pi / 2} K(x, t)\left(\Phi\left(t+\frac{\pi}{2}\right)+\Phi\left(\frac{\pi}{2}-t\right)\right) d t+\frac{1}{2} \int_{\pi / 2}^{x} K(x, t) \Phi\left(t-\frac{\pi}{2}\right) d t\right] \\
& \quad+\frac{1}{2} \Phi\left(x+\frac{\pi}{2}\right)+\frac{1}{2} \int_{\pi / 2}^{x} K(x, t) \Phi\left(t+\frac{\pi}{2}\right) d t=0
\end{aligned}
$$

Так как $\Phi(x)$ уже определена (из (15)) при $0 \leqslant x \leqslant \pi$, то в квадратных скобках в (16) записана известная функция, и это уравнение типа Вольтерра на функцию $\tilde{\Phi}(x)=\frac{1}{2} \Phi\left(x+\frac{\pi}{2}\right)$, определяющее $\Phi(x)$ при $\pi \leqslant x \leqslant \frac{3}{2} \pi$. Продолжая действовать таким образом, мы получим $\Phi(x)$ на всем интересуюшем нас отрезке и докажем ее аналитичность. Лемма доказана.

Теперь мы готовы перейти к описанию функций $K(x, y)$ и $\Phi(x)$, отвечающих операторам класса $S$. Запишем уравнение (12) в виде

$$
\begin{aligned}
\frac{1}{2}[\Phi(x+y)+\Phi(x-y)] & +K(x, y)+\frac{1}{2} \int_{0}^{y} K(x, t)[\Phi(t+y)+\Phi(y-t)] d t \\
& +\frac{1}{2} \int_{y}^{x} K(x, t)[\Phi(t+y)+\Phi(t-y)] d t=0, \quad 0 \leqslant y \leqslant x
\end{aligned}
$$

и продифференцируем его $n$ раз по переменной $y$. Получим:

$$
\begin{aligned}
& \frac{1}{2}\left[\Phi^{(n)}(x+y)+(-1)^{n} \Phi^{(n)}(x-y)\right]+\frac{\partial^{n} K(x, y)}{\partial y^{n}}+\Phi^{\prime}(0) \frac{\partial^{n-2} K(x, y)}{\partial y^{n-2}} \\
& +\Phi^{\prime \prime \prime}(0) \frac{\partial^{n-4} K(x, y)}{\partial y^{n-4}}+\cdots+\Phi^{\left(2\left[\frac{n}{2}\right]-1\right)}(0) \frac{\partial^{\left(n-2\left[\frac{n}{2}\right]\right)} K(x, y)}{\partial y^{\left(n-2\left[\frac{n}{2}\right]\right)}} \\
& +\frac{1}{2} \int_{0}^{y} K(x, t)\left[\Phi^{(n)}(t+y)+\Phi^{(n)}(y-t)\right] d t \\
& +\frac{1}{2} \int_{y}^{x} K(x, t)\left[\Phi^{(n)}(t+y)+(-1)^{n} \Phi^{(n)}(t-y)\right] d t=0 .
\end{aligned}
$$

Сразу отметим, что если в уравнениях (17) при $n$ нечетных положить $y=0$, то немедленно имеем, что

$$
\left.\frac{\partial^{2 k+1} K(x, y)}{\partial y^{2 k+1}}\right|_{y=0} \equiv 0, \quad k=0,1, \ldots,
$$

т.е. разложение ядра оператора преобразования по степеням $y$ содержит только четные степени $y$.

Проинтегрировав по частям представление (9), можно получить разложение (5) для $\varphi(x, \lambda)$ и, сравнив полученные коэффициенты, записать

$$
-k_{j}(x)=\left.(-1)^{\frac{j(j+1)}{2}} \frac{\partial^{j-1} K(x, t)}{\partial t^{j-1}}\right|_{t=x} .
$$


Здесь использованы равенства (18). Из (19) и из известных свойств цепочки функций $k_{j}(x)$ следует, что если для некоторого $j_{0}$ функция $\left.\frac{\partial^{j_{0}} K(x, t)}{\partial t^{j_{0}}}\right|_{t=x} \equiv 0$, то такое же тождество верно и для всех $j>j_{0}$, и так как в этом случае по леммам 1 и 2 функция $K(x, t)$ - аналитическая, то нетрудно вывести утверждение следующей леммы.

Лемма 3. Ядро оператора преобразования $K(x, t)$ для операторов класса S есть полином по второй переменной.

Вернемся к уравнениям (17) и рассмотрим их при $n=0,2,4, \ldots$ При $n=0$ это будет исходное уравнение $(12)$, выразим из него $K(x, y)$ и подставим в $(17)$ при $n=2$ :

$$
\frac{\partial^{2} F}{\partial y^{2}}-\Phi^{\prime}(0) F+\frac{\partial^{2} K}{\partial y^{2}}+\int_{0}^{x} K(x, t)\left[\frac{\partial^{2} F}{\partial y^{2}}-\Phi^{\prime}(0) F\right] d t=0 .
$$

Выразив отсюда $\frac{\partial^{2} K}{\partial y^{2}}$ и подставив в $(17)$ при $n=4$, получим

$$
\begin{aligned}
& \frac{\partial^{4} F}{\partial y^{4}}-\Phi^{\prime}(0) \\
& \frac{\partial^{2} F}{\partial y^{2}}+\left[\Phi^{\prime 2}(0)-\Phi^{\prime \prime \prime}(0)\right] F+\frac{\partial^{4} K}{\partial y^{4}} \\
& +\int_{0}^{x} K(x, t)\left[\frac{\partial^{4} F}{\partial y^{4}}-\Phi^{\prime}(0) \frac{\partial^{2} F}{\partial y^{2}}+\left[\Phi^{\prime 2}(0)-\Phi^{\prime \prime \prime}(0)\right] F\right] d t=0 .
\end{aligned}
$$

Вообще, при $n=2 k$ повторением этой процедуры будем иметь

$$
M_{2 k, y}(F(x, y))+\frac{\partial^{2 k} K(x, y)}{\partial y^{2 k}}+\int_{0}^{x} K(x, t) M_{2 k, y}(F(t, y)) d t=0
$$

где $M_{2 k, y}$ - линейное дифференциальное выражение с постоянными коэффициентами (полиномами от значений нечетных производных $\Phi(x)$ в нуле) порядка $2 k$ по переменной $y$, содержащее производные только четного порядка. Пусть $\frac{\partial^{2 k} K(x, y)}{\partial y^{2 k}} \equiv 0$ при некотором $k$. Рассмотрим уравнение $(20)$ при этом $k$ и положим там значение параметра $y=0$ :

$$
M_{2 k, x}(\Phi(x))+\int_{0}^{x} K(x, t) M_{2 k, t}(\Phi(t)) d t=0 .
$$

Это однородное уравнение Вольтерра относительно функции $M_{2 k, x}(\Phi(x))$ и, значит, имеет лишь нулевое решение:

$$
M_{2 k, x}(\Phi(x))=0
$$

Таким образом, если оператор принадлежит классу $S$, то его переходная функция обратной за дачи при некотором натуральном $k$ (и при всех последующих) удовлетворяет уравнению (22). Обратно, если при некотором $k$ выполнено (22), то, как следует из $(20), \frac{\partial^{2 k} K(x, y)}{\partial y^{2 k}} \equiv 0$ и ядро оператора преобразования $K(x, y)$ есть полином по второй переменной, что по лемме 3 означает принадлежность оператора классу $S$. Таким образом, уравнения $(22)$ полностью описывают класс $S$. 
ЗАмечАниЕ. Так как (22) есть линейное уравнение с постоянными коэффициентами, то $\Phi(x)$ есть обязательно квазиполином.

Покажем, как в случае оператора класса $S$ решить основное интегральное уравнение обратной задачи. Снова обратимся к уравнениям (17) при четных $n$, положим в них $y=0$ и подставим неизвестную функцию $K(x, y)$ в виде $K(x, y)=$ $\sum_{m=0}^{N} q_{m}(x) y^{2 m}$. Получим

$$
\begin{aligned}
& \Phi(x)+a_{11} q_{0}(x)+\sum_{m=0}^{N} q_{m}(x) \int_{0}^{x} t^{2 m} \Phi(t) d t=0, \\
& \Phi^{\prime \prime}(x)+a_{21} q_{0}(x)+a_{22} q_{1}(x)+\sum_{m=0}^{N} q_{m}(x) \int_{0}^{x} t^{2 m} \Phi^{\prime \prime}(t) d t=0, \\
& \Phi^{(2 N)}(x)+a_{N+1,1} q_{0}(x)+\cdots+a_{N+1, N+1} q_{N}(x)+\sum_{m=0}^{N} q_{m}(x) \int_{0}^{x} t^{2 m} \Phi^{(2 N)}(t) d t=0,
\end{aligned}
$$

где $a_{i j}$ - числа, зависящие только от $\Phi(x)$, конкретный вид которых опустим. Мы получили линейную систему для неизвестных функций $q_{0}(x), \ldots, q_{N}(x)$, определяющих $K(x, y)$. Заметим, что так как $\Phi(x)$ - квазиполином, то $\Phi(x)$-целая функция и, значит, определитель этой системы $\Delta(x)$ - также целая функция. Если записать (в стандартных обозначениях метода Крамера) $q_{i}(x)=\frac{\Delta_{i}(x)}{\Delta(x)}$ и найти вид $\Delta(x)$ и $\Delta_{i}(x)$, то можно увидеть, что $\Delta^{\prime}(x)=-\Delta_{0}(x)-x^{2} \Delta_{1}(x)-\cdots-x^{2 N} \Delta_{N}(x)$ и отсюда

$$
K(x, x)=\sum_{m=0}^{N} q_{m}(x) x^{2 m}=-\frac{\Delta^{\prime}(x)}{\Delta(x)} .
$$

Мы видим, что $K(x, x)$ может иметь в качестве особенностей только полюса первого порядка, а $q(x)=2 \frac{d}{d x} K(x, x)$ в окрестности любой своей особой точки обязательно имеет вид $q(x)=\frac{\text { const }}{(x-a)^{2}}+\tilde{q}(x)$, где $\tilde{q}(x)$ - регулярная в окрестности точки $a$ функция. Эти замечания сушественно уточняют результат леммы 1 .

Полученное нами характеристическое уравнение (22) в общем виде чрезвычайно сложно для исследования, так как записьваемое формально линейньм уравнением с постоянными коэффициентами, оно является по сути существенно нелинейной задачей из-за того, что постоянные коэффициенты нелинейно связаны со значениями в точке интересующего нас решения и его производных. Однако один частньй случай поддается исследованию, и соответствующий результат мы сформулируем в виде леммы.

ЛЕмма 4. Любой полином по четным степеням $x$ является переходной функиией обратной задачи для некоторого оператора класса $S[0, a]$ с некоторым $a>0$.

ДокАЗАТЕЛьство. Действительно, возьмем $\Phi(x)=\sum_{n=0}^{l} a_{n} x^{2 n}$. Тогда $\Phi^{(2 l+1)}(0)=0$ и $M_{2 l+2, x}(\Phi(x))=\Phi^{(2 l+2)}(x)=0$ и для доказательства леммы осталось показать, что сушествует отрезок $[0, a]$, на котором основное интегральное уравнение обратной задачи (12)-(13) с выбранной нами функцией $\Phi(x)$ 
разрешимо. Но это уравнение Фредгольма второго рода с непрерывным ядром $F(x, y)$, и на достаточно малом отрезке изменения $x$ норма этого ядра будет меньше единицы и решение явно предъявляется рядом Неймана. Лемма доказана.

3. Приближенное вычисление первых собственных чисел операторов класса $S$. Основньм результатом этого пункта является следующая

TEOPEMA 1. Пусть $\left\{\lambda_{n}\right\}_{n=0}^{\infty}-$ cпектр некоторого оператора $L$ из класса $S$ u nyсmb

$$
\sum_{n=0}^{\infty}\left(\lambda_{n}^{k}-A_{n}(k)\right)=B(k), \quad k=1,2, \ldots,
$$

- полная система регуляризованных следов этого оператора. Тогда система (24) однозначно определяет спектр $\left\{\lambda_{n}\right\}_{n=0}^{\infty}$ u, более того, для любого $\varepsilon>0$ существует натуральное $N(\varepsilon)$, а также существует натуральное $K$, зависящее только от оператора $L$ ( и не зависящее от $\varepsilon$ ), что если использовать в $A_{n}(k)(24)$ при $k=1, \ldots, K$ первье $N$ членов асимптотического разложения $\lambda_{n}$ по степеням $n$, то будут верны неравенства

$$
\left|\sum_{n=0}^{K-1}\left(\lambda_{n}^{k}-A_{n}(k)\right)-B(k)\right| \leqslant \varepsilon, \quad k=1, \ldots, K .
$$

Причем мохно взять $K=13+\left[12 \pi(3+H) 2^{p} M_{1}\right], a N \geqslant 2(K-1) \times$ $\lg (4 K+12)-\lg \frac{10 \varepsilon}{3}$. Здесь $p-$ число ненулевых членов асимптотики (5) для $L, M_{1}=\max _{0 \leqslant i \leqslant p}\left\{\max _{x \in[0, \pi]}\left(\frac{1}{2} \int_{0}^{x} q(x)+h\right)^{(i)}\right\}$.

ДокАЗАтЕльство. Уравнение на собственные числа оператора (3)-(4) имеет вид $\varphi^{\prime}(\pi, \lambda)+H \varphi(\pi, \lambda)=0$, что для оператора класса $S$ можно (в силу конечности суммы (5)) переписать в виде

$$
e^{2 i \pi \sqrt{\lambda}} \sum_{k=0}^{p} \frac{a_{k}}{(\sqrt{\lambda})^{k}}-\sum_{k=0}^{p} \frac{b_{k}}{(\sqrt{\lambda})^{k}}=0
$$

с некоторыми постоянными коэффициентами $a_{k}, b_{k}$, выражающимися через коэффициенты $k_{j}(x)(5)$. Индекс $p$ в (26) совпадает с номером последнего ненулевого коэффициента в (5). Оценим коэффициенты $a_{k}, b_{k}$ в $(26)$, для чего необходима оценка $k_{j}(x)$ и $k_{j}^{\prime}(x)$ на отрезке $[0, \pi]$. Из формул $(6)$ видно, что для оценки $k_{p}^{\prime}(x)$ необходимо оценить $k_{p-1}^{\prime \prime}(x)$, для чего необходима оценка $k_{p-2}^{\prime \prime \prime}(x)$ и т. д.

Заметим, что введенная в формулировке теоремы константа $M_{1}$ может быть записана так:

$$
M_{1}=\max _{0 \leqslant i \leqslant p}\left\{\max _{x \in[0, \pi]}\left|k_{1}^{(i)}(x)\right|\right\} .
$$

Так как $k_{1}(x)=\frac{1}{2} \int_{0}^{x} q(x) d x+h$, то все необходимые нам производные $q(x)$ не превосходят $2 M_{1}$. Из (6) легко получаем, что $\forall l \in \mathbb{N}$

$$
k_{2}^{(l+1)}(x)=\frac{1}{2}\left[k_{1}^{(l+2)}(x)-\sum_{m=0}^{l} C_{m}^{l} q^{(m)}(x) k_{1}^{(l-m)}(x)\right]
$$


и для любого $l=0, \ldots, p-2$

$$
\left|k_{2}^{(l+1)}(x)\right| \leqslant \frac{1}{2} M_{1}+M_{1} M_{1} 2^{l} \leqslant 2^{p-1} M_{1}^{2} .
$$

Для самой функции $k_{2}(x)$ воспользуемся равенством

$$
k_{2}(x)=-\frac{1}{2} k_{1}^{\prime}(0)+\frac{1}{2} k_{1}^{\prime}(x)-\frac{1}{2} \int_{0}^{x} q(x) k_{1}(x) d x
$$

и увидим, что все интересующие нас производные $k_{2}(x)$, включая нулевую производную, оцениваются константой $M_{2}=\pi 2^{p-1} M_{1}^{2}$. Обозначим через $M_{j}$ константу, ограничивающую все нужные нам производные $k_{j}(x)$. Тогда повторением указанной процедуры нетрудно получить следующую оценку: $M_{j}=\pi^{j-1} 2^{(j-1) p-\frac{j(j+1)}{2}} M_{1}^{j}$. Для упрощения дальнейших выкладок примем:

$$
\max _{0 \leqslant i \leqslant p+1-l}\left\{\max _{x \in[0, \pi]}\left|k_{l}^{(i)}(x)\right|\right\} \leqslant \pi^{l} 2^{l p} M_{1}^{l}
$$

Далее, легко установить, что коэффициенты $a_{k}, b_{k}, k=1, \ldots, p$, в (26) (при нормировке $a_{0}=b_{0}=1$ ) образуются как сумма не более чем трех слагаемых, каждое из которых есть значение в точке $\pi$ функций $k_{k}(x), k_{k-1}(x)$ или их первых производных, причем одно из этих слагаемых умножено на $H$.

Исходя из этого и (28), запишем

$$
\left|a_{k}\right|,\left|b_{k}\right| \leqslant(2+H) \pi^{k} 2^{k p} M_{1}^{k}, \quad k=1, \ldots, p .
$$

Перепишем уравнение на собственные числа $\left\{\lambda_{n}\right\}(26)$ в виде

$$
e^{2 i \pi \sqrt{\lambda_{N}}}=\left(1+\sum_{k=1}^{p} \frac{b_{k}}{\left(\sqrt{\lambda_{n}}\right)^{k}}\right)\left(1+\sum_{k=1}^{p} \frac{a_{k}}{\left(\sqrt{\lambda_{n}}\right)^{k}}\right)^{-1}
$$

и прологарифмируем (30) (пользуясь также классическим фактом о том, что $\left\{\lambda_{n}\right\}$ находится в окрестности именно $n \in \mathbb{N}$ ):

$$
\sqrt{\lambda_{n}}=n+\frac{1}{2 \pi i} \ln \left(1+\sum_{k=1}^{p} \frac{b_{k}}{\left(\sqrt{\lambda_{n}}\right)^{k}}\right)-\frac{1}{2 \pi i} \ln \left(1+\sum_{k=1}^{p} \frac{a_{k}}{\left(\sqrt{\lambda_{n}}\right)^{k}}\right) .
$$

Из оценок (29) легко следует, что логарифмы в (31) разлагаются в ряд Тейлора (по степеням сумм, прибавляемых к 1 ) при $\sqrt{\lambda_{n}}>(3+H) \pi M_{1} 2^{p}$, а следовательно, они разлагаются в ряд Тейлора по степеням $\frac{1}{\sqrt{\lambda_{n}}}$ в той же области:

$$
\sqrt{\lambda_{n}}=n+\sum_{k=1}^{\infty} \frac{d_{k}}{\left(\sqrt{\lambda_{n}}\right)^{k}}
$$

Опуская несложную, но весьма громоздкую процедуру разложения логарифмов из (31) по степеням $\frac{1}{\sqrt{\lambda_{n}}}$ и применения к получаемым выражениям оценок (29), запишем необходимую для дальнейшего оценку коэффициентов $d_{k}$ из $(32)$ :

$$
\left|d_{k}\right| \leqslant\left[\pi(3+H) 2^{p} M_{1}\right]^{k}, \quad k=1,2, \ldots
$$


Оценка (33) указывает, что, вообще говоря, равенства (32) верны (не как асимптотические, а как точные, со сходящимся рядом) не для всех $\lambda_{n}$, а только начиная с некоторого номера. Используя рассуждение типа обрашения степенного ряда по Бурману-Лагранжу, нетрудно заметить, что, начиная с некоторого номера $K$, имеют место представления $\sqrt{\lambda_{n}}$ в виде сходящегося ряда по степеням $n$ :

$$
\sqrt{\lambda_{n}}=n+\sum_{k=1}^{\infty} \frac{c_{k}}{n^{k}}, \quad n \geqslant K
$$

Нам необходима оценка $K$ и $c_{k}$ из $(34)$.

Итак, рассмотрим функцию ${ }^{2} \psi(z)=A+\sum_{k=0}^{\infty} a_{k} z^{k+1}$, где $A \neq 0,\left|a_{k}\right| \leqslant$ $C R^{-k-1}, C>0$ и $R>0$ - некоторые константы. Тогда функция $w(z)=\frac{z}{\psi(z)}$ аналитична в некоторой окрестности нуля и

$$
\frac{1}{w(z)}=\frac{A}{z}+\sum_{k=0}^{\infty} a_{k} z^{k}
$$

Нам надо в некоторой окрестности нуля получить разложение

$$
\frac{1}{z}=\frac{1}{A w}+\sum_{k=0}^{\infty} b_{k} w^{k}
$$

и оценить снизу $\rho$ - радиус сходимости ряда $\sum b_{k} w^{k}$. Сразу поясним, что разложение (35) соответствует (32) ( $n$ по $\left.\sqrt{\lambda_{n}}\right)$, а (36) соответствует $(34)\left(\sqrt{\lambda_{n}}\right.$ по $\left.n\right)$, и $K$ из (34) связано с $\rho$ так: $K=\left[\frac{1}{\rho}\right]+1$.

Пусть $D$ - область в $C$, содержащая нуль, $\partial D$ - простая замкнутая спрямляемая кривая, $w(z)$ аналитична в $\bar{D}$ и

$$
w\left(z_{1}\right) \neq w\left(z_{2}\right) \quad \forall z_{1}, z_{2} \in \bar{D}, \quad z_{1} \neq z_{2}
$$

Также пусть $B=\min _{z \in \partial D}|w(z)|$. Так как у нас $w(0)=0$, то в силу $(37) B>0$. Другими словами, $w$ является биекцией $\bar{D}$ на ее образ и этот образ содержит круг $K_{B}=\{w \in C|| w \mid \leqslant B\}$. Пусть $G=w^{-1}\left(K_{B}\right)$. Запишем необходимые в дальнейшем формулы для коэффициентов степенного ряда некоторой функции

$$
f(z)=f(z(w))=\sum_{n=0}^{\infty} b_{n} w^{n}
$$

определенные в $G$ :

$$
b_{n}=\frac{1}{2 \pi i} \int_{|w|=B} \frac{f(z(w))}{w^{n+1}} d w=\frac{1}{2 \pi i} \int_{\partial G} \frac{f(z) w^{\prime}(z)}{(w(z))^{n+1}} d z
$$

\footnotetext{
${ }^{2}$ Авторы признательны А. Ю. Попову за существенную помощь в получении нижеследующей оценки для $K$ и $c_{k}$.
} 
Нас в соответствии с (36) интересует разложение функции

$$
f(z)=\frac{1}{z}-\frac{1}{A w(z)}=\frac{1}{z}-\frac{\psi(z)}{A z}=\frac{A-\psi(z)}{A z},
$$

аналитической в круге $|z|<R$. В качестве областей $D$ мы можем брать круги $|z|<r, r<R$, причем $r$ надо выбрать так, чтобы $w(z)$ было инъективным в $|z|<r$, а величина

$$
B=B(r)=\min _{|z|=r}|w(z)|=\min _{|z|=r}\left|\frac{z}{\psi(z)}\right|=\frac{r}{\max _{|z|=r}|\psi(z)|}
$$

была бы возможно большей. Тогда $\rho=\sup _{r} B(r)$.

Лемма 5. Пусть $C_{1}=\frac{C}{|A|}, r_{0}=\frac{R}{2\left(1+C_{1}\right)}$. Тогда $\psi(z) \neq 0$ при $|z|<2 r_{0} u$ в этом круге

$$
w(z)=\sum_{k=1}^{\infty} \delta_{k} z^{k}, \quad\left|\delta_{k}\right| \leqslant \frac{1+2 C_{1}}{C+|A|} r_{0}^{1-k}, \quad k \geqslant 2
$$

ДокАЗАТЕЛЬСТво. Имеем

$$
|\psi(z)| \geqslant|A|-\sum_{k=0}^{\infty} C\left(\frac{|z|}{R}\right)^{k+1}=|A|\left(1-\frac{C_{1}|z|}{R-|z|}\right) .
$$

Если $|z|<\frac{R}{1+C_{1}}$, то $\frac{C_{1}|z|}{R-|z|}<1$ и, действительно, $\psi(z) \neq 0$ при $|z|<2 r_{0}$. Если $|z|=r_{0}$, то $|\psi(z)| \geqslant|A| \frac{1+C_{1}}{2 C_{1}+1}$ и

$$
\left|\delta_{k}\right|=\left|\frac{1}{2 \pi i} \int_{|z|=r_{0}} \frac{w(z)}{z^{k+1}} d z\right| \leqslant \frac{\max _{|z|=r_{0}}\left|\frac{z}{\psi(z)}\right|}{r_{0}^{k}}=\frac{r_{0}^{1-k}}{\min _{|z|=r_{0}}|\psi(z)|} \leqslant \frac{2 C_{1}+1}{|A|\left(1+C_{1}\right)} r_{0}^{1-k} .
$$

Лемма доказана.

ЛЕмма 6. Если $\sum_{n=2}^{\infty} n\left|\delta_{n}\right| r^{n-1}<\left|\delta_{1}\right|$, то отображсение $w(z)=\sum_{n=1}^{\infty} \delta_{n} z^{n}$ ингективно в круге $|z| \leqslant r$.

ДоказАТЕЛьство. Имеем

$$
w\left(z_{1}\right)-w\left(z_{2}\right)=\delta_{1}\left(z_{1}-z_{2}\right)+\sum_{n=2}^{\infty} \delta_{2}\left(z_{1}^{n}-z_{2}^{n}\right)
$$

Следовательно, при $z_{1} \neq z_{2},\left|z_{1}\right|,\left|z_{2}\right| \leqslant r$

$$
\left|\frac{w\left(z_{1}\right)-w\left(z_{2}\right)}{z_{1}-z_{2}}\right| \geqslant\left|\delta_{1}\right|-\sum_{n=2}^{\infty}\left|\delta_{n}\right|\left|\sum_{j=0}^{n-1} z_{1}^{j} z_{2}^{n-1-j}\right| \geqslant\left|\delta_{1}\right|-\sum_{n=2}^{\infty} n\left|\delta_{n}\right| r^{n-1}>0 .
$$

Лемма доказана. 
$\mathrm{y}$ нас $\delta_{1}=1 / A$ и надо подобрать $r$ так, чтобы было выполнено неравенство

$$
\frac{1}{|A|} \frac{1+2 C_{1}}{1+C_{1}} \sum_{k=2}^{\infty} k\left(\frac{r}{r_{0}}\right)^{k-1}<\frac{1}{|A|}
$$

или

$$
\sum_{k=2}^{\infty} k\left(\frac{r}{r_{0}}\right)^{k-1}<\frac{1+C_{1}}{1+2 C_{1}}
$$

Очевидно, достаточно выполнения неравенства $\frac{1}{\left(1-\left(r / r_{0}\right)\right)^{2}}-1 \leqslant 1 / 2$, и отсюда $r / r_{0} \leqslant 1-\sqrt{2 / 3}$. Примем $r=\frac{2}{11} r_{0}=\frac{R}{11\left(1+C_{1}\right)}$ и оценим $\rho$. В силу изложенного перед леммой $5 \rho \geqslant \frac{r}{\max _{|z|=r}|\psi(z)|}$. В отношении $\max |\psi(z)|$ имеем:

$$
\max _{|z|=r}|\psi(z)| \leqslant|A|+\sum_{k=0}^{\infty}\left|a_{k}\right| r^{k+1} \leqslant|A|+C \frac{r}{R} \sum_{k=0}^{\infty}\left(\frac{r}{R}\right)^{k}=|A|+\frac{r C}{R-r}
$$
и окончательно для $\rho$ получаем следуюшую оценку: $\rho \geqslant \frac{R}{12(|A|+C)}$ и для $K$
верно:

$$
K=\left[\frac{12(|A|+C)}{R}\right]+1 .
$$

Наконец, воспользуемся разложением (32) и оценками (33) для записи оценки для $K$ в терминах, прямо связанных с исходной задачей: $A=1$, $C=\frac{1}{\pi(3+H) 2^{p} M_{1}}, R=C$, и имеем

$$
K=\left[12+12 \pi(3+H) 2^{p} M_{1}\right]+1=13+\left[12 \pi(3+H) 2^{p} M_{1}\right] .
$$

Для оценки коэффициентов $c_{k}$ из (34) используем формулы (38), оценки для $w(z)$ из леммы 5 и несложную оценку максимума $\psi(z)$. Итак,

$$
\begin{aligned}
\left|c_{k}\right| & \leqslant\left|\frac{1}{2 \pi i} \int_{|z|=\frac{2}{11} r_{0}} \frac{A-\psi(z)}{A z} \frac{w^{\prime}(z)}{(w(z))^{k+1}} d z\right| \\
& =\left|\frac{1}{2 \pi i} \int_{|z|=\frac{2}{11} r_{0}} \frac{1}{A}\left(\sum_{l=0}^{\infty} a_{l} z^{l}\right) \frac{(\psi(z))^{k+1}}{z^{k+1}}\left(\sum_{l=1}^{\infty} \delta_{l} l z^{l}-1\right) d z\right| \\
& \leqslant 24\left(\frac{6}{5} \pi(3+H) 2^{p} M_{1}\right)^{k} .
\end{aligned}
$$

Наконец, докажем неравенства (25). Мы опять пожертвуем качеством оценки (они будут весьма грубыми) в пользу простых и коротких рассуждений и выкладок. Итак, пусть номер $N$ таков, что

$$
\left|\sqrt{\lambda_{K}}-A_{N}(K)\right|<\varepsilon_{K}(N),
$$


где $A_{N}(K)$ отрезок разложения $(34)$ до члена $\frac{C_{N}}{K^{N}}$. Подберем для любого $\varepsilon>0$ номер $N$ так, чтобы

$$
\left|\left(\sqrt{\lambda_{K}}\right)^{l}-A_{N}^{l}(K)\right|<\varepsilon
$$

для $l=1,2 \ldots, 2(K-1)$. Из (34) и оценок $(40),(41)$ легко получаем

$$
\left|\sqrt{\lambda_{K}}\right|<K+3
$$

и, делая оценку $\left|A_{N}(K)\right|<2\left|\sqrt{\lambda_{k}}\right|,(1+\varepsilon)^{n}-1<\varepsilon n 2^{n-1}$, имеем, что при выборе

$$
\varepsilon_{K}(N)<\frac{\varepsilon}{(4(K+3))^{2(K-1)}}
$$

из (42) будет следовать (43). Далее, так как

$$
\varepsilon_{K}(N)=\sum_{l=N+1}^{\infty} \frac{C_{l}}{K^{l}}
$$

то, выбрав $N$ в соответствии с неравенством

$$
\frac{3}{10^{N+1}} \leqslant \frac{\varepsilon}{(4(K+3))^{2(K-1)}}
$$

мы определим $A_{N}(K)$ так, что будет выполнено (45). Теорема 1 доказана.

4. Приближение произвольного оператора Штурма-Лиувилля оператором класса $S$.

ТЕОРема 2. Для произвольного оператора Штурма-Лиувилля L с потенииалом из $L_{2}[0, \pi]$ и произвольного $\varepsilon>0$ существует оператор класса $S$ с областью определения такой жсе, как и у L, и такой, что норма их разности не превосходит $\varepsilon$.

ДоказАтЕльство. Рассмотрим оператор (3), (4) и его переходную функцию обратной задачи $\Phi(x)(11)$. Если $q(x) \in L_{2}[0, \pi]$, то $\Phi(x) \in A C[0,2 \pi](\Phi(x)$ может иметь в точке $2 \pi$ разрыв первого рода, что несушественно для дальнейшего, и мы будем принимать за $\Phi(x)$ функцию, совпадающую с $\Phi(x)$ на $[0,2 \pi)$ и равную в $x=$ $2 \pi$ пределу $\Phi(x))$, и $\Phi^{\prime}(x) \in L_{2}[0,2 \pi]$. Приблизим $\Phi(x)$ многочленом $P \Phi\left(x, \varepsilon_{1}\right)$, содержащим лиш четные степени $x$, с точностью до $\varepsilon_{1}$ по норме $C[0,2 \pi]$ и таким, что $P \Phi^{\prime}\left(x, \varepsilon_{1}\right)$ приближает $\Phi^{\prime}(x)$ в $L_{2}[0,2 \pi]$ также с точностью $\varepsilon_{1}$ (о способах построения подобных приближений см. [13]). Тогда, очевидно, функция

$$
P F\left(x, y, \varepsilon_{1}\right)=\frac{1}{2}\left[P \Phi\left(x+y, \varepsilon_{1}\right)+P \Phi\left(|x-y|, \varepsilon_{1}\right)\right]
$$

будет аналогично приближать $F(x, y)(13)$.

Основное интегральное уравнение обратной задачи (12) есть уравнение по переменной $y$ с параметром $x$, и при каждом фиксированном $x$ есть уравнение Фредгольма второго рода. Обозначая оператор

$$
\int_{0}^{x} K(x, t) F(t, y) d t=\mathscr{F}_{x}(K(x, y)),
$$


перепишем (12) в более удобной для нас форме:

$$
\left(I+\mathscr{F}_{x}\right) K(x, y)=-F(x, y) .
$$

Аналогичное уравнение с $P F\left(x, y, \varepsilon_{1}\right)$ вместо $F(x, y)$ запишем так:

$$
\left(I+\mathscr{P} \mathscr{F}_{x, \varepsilon_{1}}\right) K_{\varepsilon_{1}}(x, y)=-P F\left(x, y, \varepsilon_{1}\right) .
$$

Считая для удобства $P F(x, y, 0)=F(x, y)$, перепишем (48) в виде

$$
\left(I+\mathscr{F}_{x}+\left[\mathscr{P}_{x, \varepsilon_{1}}-\mathscr{F}_{x}\right]\right) K_{\varepsilon_{1}}(x, y)=-F(x, y)+\left(-P F\left(x, y, \varepsilon_{1}\right)+F(x, y)\right) .
$$

Так как $\Phi(x)$ является переходной функцией обратной задачи некоторого оператора $L$, то уравнение (47) однозначно разрешимо при любом $x[9]$ и, следовательно, существует оператор $\left(I+\mathscr{F}_{x}\right)^{-1}$. Применим этот оператор к обеим частям равенства (49):

$$
\begin{aligned}
\left(I+\left(I+\mathscr{F}_{x}\right)^{-1}\left(\mathscr{P} \mathscr{F}_{x, \varepsilon_{1}}-\mathscr{F}_{x}\right)\right) K_{\varepsilon_{1}}(x, y) \\
=K(x, y)+\left(I+\mathscr{F}_{x}\right)^{-1}\left(F(x, y)-P F\left(x, y, \varepsilon_{1}\right)\right)
\end{aligned}
$$

Далее, так как $P F\left(x, y, \varepsilon_{1}\right)$ есть равномерное приближение для $F(x, y)$, то норма оператора $\mathscr{P} \mathscr{F}_{x, \varepsilon_{1}}-\mathscr{F}_{x}$ может быть выбором $\varepsilon_{1}$ сделана сколь угодно малой (оценку опустим ввиду тривиальности), а значит, и норма $\left(I+\mathscr{F}_{x}\right)^{-1}\left(\mathscr{P} \mathscr{F}_{x, \varepsilon_{1}}-\mathscr{F}_{x}\right)$ тоже может быть сделана сколь угодно мала. Тогда оператор, применяемый к $K_{\varepsilon_{1}}(x, y)$ в $(50)$, обратим и, более того, обратный к нему представим в виде суммы ряда Неймана. Применим этот ряд Неймана к левой и правой частям (50):

$$
\begin{aligned}
K_{\varepsilon_{1}}(x, y)= & \left(\sum_{l=0}^{+\infty}(-1)^{l}\left(\left(I+\mathscr{F}_{x}\right)^{-1}\left(\mathscr{P} \mathscr{F}_{x, \varepsilon_{1}}-\mathscr{F}_{x}\right)\right)^{l}\right) \\
& \times\left(K(x, y)+\left(I+\mathscr{F}_{x}\right)^{-1}\left(F(x, y)-P F\left(x, y, \varepsilon_{1}\right)\right)\right) \\
= & K(x, y)-\left(I+\left(I+\mathscr{F}_{x}\right)^{-1}\left(\mathscr{P}_{x, \varepsilon_{1}}-\mathscr{F}_{x}\right)\right) \\
& \times\left(I+\mathscr{F}_{x}^{-1}\right)\left(F(x, y)-P F\left(x, y, \varepsilon_{1}\right)+\left(\mathscr{P} \mathscr{F}_{x, \varepsilon_{1}}-\mathscr{F}_{x}\right) K(x, y)\right) .
\end{aligned}
$$

Из (51) уже легко оценивается $\left|K_{\varepsilon_{1}}(x, y)-K(x, y)\right|$. Заметим, что попутно мы доказали разрешимость основного интегрального уравнения, основанного на функции $P \Phi\left(x, \varepsilon_{1}\right)$, что само по себе не очевидно, так как про эту функцию заранее не известно, что она является переходной функцией некоторого оператора Штурма-Лиувилля на отрезке $[0, a]$ (ср. с леммой 4).

Дифференцируя уравнение (12) по $x$ и по $y$, соответственно получим:

$$
\begin{aligned}
K_{x}^{\prime}(x, y)+F_{x}^{\prime}(x, y)+ & K(x, x) F(x, y)+\int_{0}^{x} K_{x}^{\prime}(x, t) F(t, y) d t=0 \\
K_{y}^{\prime}(x, y)+F_{y}^{\prime}(x, y)+ & K(x, x) F(x, y) \\
& -K(x, 0) \Phi(y)-\int_{0}^{x} K_{t}^{\prime}(x, t) F(t, y) d t=0 .
\end{aligned}
$$

Совершенно аналогично тому, как было получено уравнение (51), мы можем исследовать и (52), (53) (используя уже имеющуюся из (51) функцию $K_{\varepsilon_{1}}(x, y)$ 
и ее близость к $K(x, y))$ и доказать возможность дифференцирования $K(x, y)$ с сохранением необходимого приближения.

Таким образом, $q_{\varepsilon_{1}}(x)=2 \frac{d}{d x} K_{\varepsilon_{1}}(x, x)$ и $h_{\varepsilon_{1}}=K_{\varepsilon_{1}}(0,0)$ будут параметрами оператора, приближающего исходньй с любой точностью, и при этом, ввиду того, что $P \Phi\left(x, \varepsilon_{1}\right)$ есть многочлен по четным степеням $x$, по лемме 4 это будут параметры оператора класса $S$. Для завершения доказательства теоремы осталось показать, что $h_{\varepsilon_{1}}$ всегда можно выбрать равньп $h$. Действительно, $h=K(0,0)=-\Phi(0)$ и мы всего лишш должны при приближении $\Phi(x)$ следить за тем, чтобы $\Phi(0)=P \Phi\left(0, \varepsilon_{1}\right)$. Теорема доказана.

В заключение заметим, что, конечно, для произвольного оператора $L$ нельзя рассчитывать на точно известную $\Phi(x)$. Саму $\Phi(x)$ можно найти приближенно последовательньм решением двух уравнений Вольтерра второго рода: сначала уравнения (14), а затем (15) и (16). Последовательные приближения для уравнений этого типа сходятся очень быстро, и получить необходимые оценки здесь несложно. То, что мы будем знать $\Phi(x)$ приближенно, на доказательстве теоремы 2 не отразится.

\section{Список литературы}

1. Дородницын A. А. Асимптотические законы распределения собственных значений для некоторых особых видов дифференциальных уравнений второго порядка // УМН. 1952. T. 7. №6. С. 3-96.

2. Гельфанд И. М., Левитан Б. М. Об одном простом тождестве для собственных значений дифференциального оператора второго порядка // Докл. АН СССР. 1953. Т. 88. C. 593-596.

3. Гельфанд И. М. О тождествах для собственных значений дифференциального оператора второго порядка // УМН. 1956. Т. 11. №1. С. 191-198.

4. Дикий Л. А. Дзета-функция обыкновенного дифференциального уравнения на конечном отрезке // Изв. АН СССР. Сер. матем. 1955. Т. 19. № 4. С. 187-200.

5. Лидский В. Б., Садовничий $В$. А. Регуляризованные суммы корней одного класса целых функций // Функц. анализ и его прилож. 1967. Т. 1. № 2. С. 52-59.

6. Лидский В.Б., Садовничий В.А. Формулы следов в случае уравнения Орра-Зоммерфельда // Изв. АН СССР. Сер. матем. 1968. Т. 32. №3. С. 633-648.

7. Дикий Л. А. Новый способ приближенного вычисления собственных чисел задачи Штурма-Лиувилля // Докл. АН СССР. 1957. Т. 116. №1. С. 12-14.

8. Шкарин C. А. О способе Гельфанда-Дикого вычисления первых собственных значений оператора Штурма-Лиувилля // Вестник МГУ. Сер. 1. Матем., мех. 1996. № 1. С. 39-44.

9. Садовничий B. A., Подольский B. E. О вычислении первых собственных значений оператора Штурма-Лиувилля // Докл. АН. 1996. Т. 346. № 2. С. 162-164.

10. Марченко В.А. Некоторые вопросы теории одномерных линейных диффференциальных операторов второго порядка. I // Труды ММО. 1952. Т. 1. С. 327-420.

11. Bummux $\Gamma$. Новейшие исследования по однозначньм аналитическим функциям. М.: Физматгиз, 1960.

12. Левитан Б. М. Обратные задачи Штурма-Лиувилля. М.: Наука, 1984.

13. Ахиезер Н. И. Лекции по теории аппроксимации. М.: Наука, 1965.

Московский государственный

Поступила в редакцию

университет им. М.В. Ломоносова

16.04.1997 